INFLUENCE OF LEAFLET'S MATRIX STIFFNESS AND FIBER ORIENTATION ON THE OPENING DYNAMICS OF A PROSTHETIC TRILEAFLET HEART VALVE

- Electronic version of an article published in [Journal of Mechanics in Medicine and Biology, Vol. 17, No. 6 (2017) 1750096 (17 pages)] [Article DOI:

10.1142/S0219519417500968] @ [copyright World Scientific Publishing Company] [Journal URL:

http://www.worldscientific.com/do i/pdf/10.1142/S021951941750096 8] 


\title{
INFLUENCE OF LEAFLET'S MATRIX STIFFNESS AND FIBER ORIENTATION ON THE OPENING DYNAMICS OF A PROSTHETIC TRILEAFLET HEART VALVE
}

\author{
ANDREA AVANZINI* \\ *Department of Mechanical and Industrial Engineering, \\ University of Brescia, Via Branze, 38, I-25123, Brescia, Italy \\ andrea.avanzini@unibs.it
}

\begin{abstract}
Biological valves are employed for aortic valve substitution since a long time but there is a growing effort toward the development of new engineered tissues, in which the complex mechanical response of native leaflets is replicated using composite materials consisting of a soft matrix with embedded reinforcing fibers. The main goal of the present study is to investigate the influence that variations on fiber orientation and matrix stiffness may have on valve dynamics.

To this aim a Fluid-Structure Interaction (FSI) model of a trileaflet valve was implemented in which the opening phase was simulated and leaflet matrix stiffness and fiber orientation were varied in the framework of an anisotropic hyperelastic strain energy function.

Results show that both parameters may affect significantly transvalvular pressure gradient and effective orifice area (EOA). For the opening phase of the valve examined less favourable flow conditions were found when preferred fiber orientation is circumferential, due to lower maximum EOA achievable. Such configuration in combination with stiffer matrix may result in significant degradation of valve performances. Overall fiber orientation can potentially be taylored to optimize valve dynamics, provided also structural aspects that may be prominent in the closure phase, are considered.
\end{abstract}


Keywords: Heart valve; FSI; Fiber reinforcement; 


\section{Introduction}

Prosthetic heart valves with trileaflet configuration provide an alternative to mechanical heart valve for aortic valve replacements, mimicking valve native geometry to improve hemodynamic performances. Many types of trileaflet valves are currently available commercially or as laboratory prototypes under investigations. Upon a structural and functional point of view, valve performances and leaflet stress and strain state are primarily governed by the design solutions adopted. Basically these may differ for presence and type of a supporting stent, leaflet shape and dimensions and leaflet/stent attachment technique. ${ }^{1}$

Quite obviously valve performances are also highly influenced by the choice of different types of biological or tissue engineered leaflets. These may exhibit different degrees of stiffness and anisotropy that could possibly be regarded as design parameters to optimize valve performances.

As an example one of the most relevant type of biological heart valves (BHV) is the pericardial valve, in which leaflets made from preserved bovine pericardial tissue are mounted on a supporting frame, after fixation treatments. Mechanical properties of chemically treated bovine pericardium (BP) such as tissue extensibility (i.e. strain level at which the tissue stiffness change) and degree of anisotropy (i.e. differences in stress-strain response parallel or perpendicular to a preferred fiber directions) are governed by local collagen fiber architecture and chemical treatment procedures. ${ }^{2,3}$ In particular stressstrain curves of BP could be altered by application of uniaxial or biaxial strains during the fixation process or by the type of chemical treatment. ${ }^{3,4}$ It has been suggested that modulating tissue anisotropy and extensibility could help achieving design objectives, such as enhancing leaflet coaptation, although, at present there is no evidence that pericardial heart valves are constructed to accommodate or take advantage of specific leaflet properties.

On the other hand there is a growing effort toward the development of tissue engineered valves and several examples can be found in literature. ${ }^{5,6}$

Biological or synthetic tissue can now be engineered in which the complex mechanical response of native tissues is replicated using composite materials consisting of a soft matrix with embedded reinforcing fibers along selected directions, potentially providing an opportunity to taylor leaflet properties to optimize valve response.

The above considerations motivated the present study in which the main goal is to investigate the potential influence that variations of leaflet mechanical properties may have on heart valve dynamics.

To this aim, among many different valve models available, a specific type of stented valve was selected, in which leaflet are mounted externally to a supporting stent. In order to carry out a realistic investigation on the influence of leaflet mechanical properties, the constitutive law and reference values of associated material parameters, were adopted basing on recent literature for pericardial valves in which anisotropic hyperelastic constitutive models were 
employed. ${ }^{7,8}$ In particular, the material of the leaflet is assumed as consisting of a soft matrix reinforced with fibers that (may) impart anisotropic properties to the mechanical response. These models essentially require the definition of a strain energy function that accounts for distinct contributions from matrix and reinforcement phases.

By varying material parameters associated with matrix stiffness and fiber orientation the influence of each constituent could then be investigated for chosen valve geometry. Similar types of constitutive law have been used also to represent valve candidate tissues other than BP. ${ }^{9}$ The results of the present study are thus intended as not necessarily restricted to pericardial valves but rather as applicable to materials of different nature.

The complex influence of anisotropic leaflet properties on valve performances was studied considering leaflet opening dynamics in the context of FluidStructure Interaction (FSI) approach. Including fluid mechanics in a valve model allows a more realistic load transfer, with the possibility to investigate simultaneously structural and functional aspects. This is especially relevant for heart valves, in which the dynamics of blood flow affect the behavior of solid components but valve structural response may alter fluid dynamics as well. Modeling the valvular structures along with a moving fluid volume is a challenging task, in particular for biological valves, in which thin-walled leaflet experiences large deformation within a fluid flow whose features are difficult to recreate computationally and not yet completely understood. Various types of FSI simulations of flexible aortic valves can be found in literature considering different types of valves with complete or selected portion of cardiac cycle. ${ }^{10-13}$

In the present study the author investigated valve response using the ALE (Arbitrary Lagrangian-Eulerian) method, as implemented in the commercial software Comsol Multiphysics (COMSOL AB, Stockholm, Sweden). Basically the blood flow is described by the Navier-Stokes equations, which provide a solution for the velocity field $\mathrm{u}_{\mathrm{f}}$ and pressure $\mathrm{p}_{\mathrm{f}}$. The coupling between solid and fluid domain is provided by the total force exerted by the fluid (caused by pressure and viscous force) on the solid boundary and by how solid displacements affects the fluid's velocity. To combine the interface between the spatial frame of the fluid and the material frame of the solid, with ALE method new mesh coordinates are computed based both on the movement of the solid's boundary and mesh smoothing of the fluid. Interested reader can refer to Comsol user guide for details of implementation. ${ }^{14}$ It should be noted that although this method is currently implemented in various commercial FEM codes of widespread use for FSI studies, its application to heart valves analyses remains difficult, especially due to high distortion of fluid mesh elements in the opening phase.

Problems deriving from excessive mesh distortion were solved by taking advantage of remeshing algorithms. 


\section{Methods}

\subsection{Computational model}

The valve under examination is a stented valve for implant in aortic position. The model consists of a solid domain (valve) and a fluid conduct (Fig.1). Note that only $1 / 6^{\text {th }}$ of the real valve was actually modelled to reduce computational effort, imposing symmetry boundary conditions on solid and fluid domain corresponding sections. Main valve dimensions (inner diameter $24.2 \mathrm{~mm}$, height $14 \mathrm{~mm}$ ) were chosen to be comparable with those of pericardial valves of this type. Leaflet shape was based on direct observations and the initial nearly closed configuration mimic the position of the leaflet in real valves before implantation. This was assumed as a stress free condition, not including any pre-stress deriving from leaflet pushing and bending operations during manufacturing.

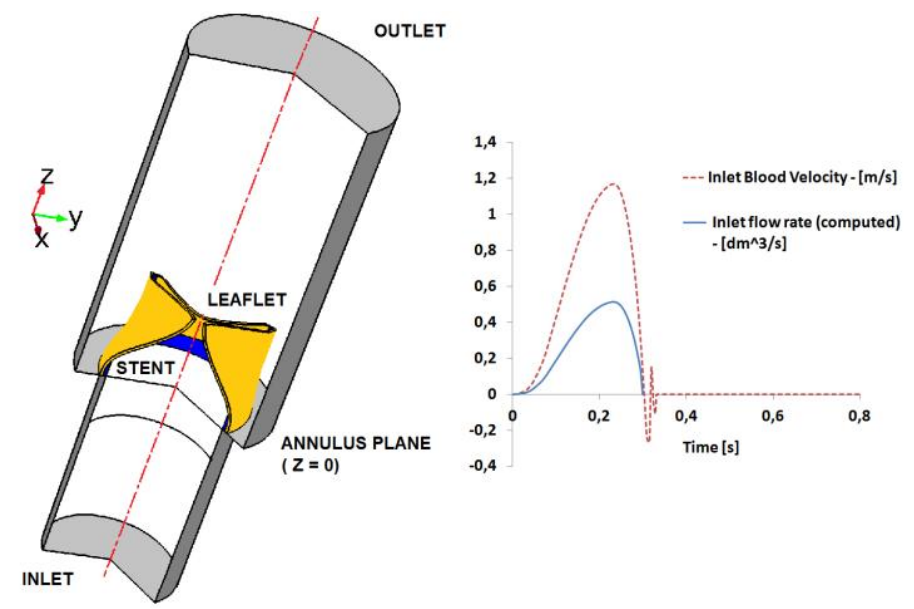

Fig. 1 Prosthetic valve, fluid conduct and inlet blood flow velocity

On the ventricular side the fluid domain consists of a straigth cylinder with the same diameter as valve inner one and lenght of $36.5 \mathrm{~mm}$. On the aortic side the diameter is $35 \mathrm{~mm}$ and the outlet section is placed at a distance of $60.75 \mathrm{~mm}$ from annulus plane, where valve ring is located and assumed to be fixed.

Fluid phase was assumed to be isothermal, incompressible, homogeneous, Newtonian and laminar, with dynamic viscosity of $0.0040 \mathrm{~Pa} \cdot \mathrm{s}$ and density of $1060 \mathrm{~kg} / \mathrm{m}^{3}$. The compliance of aortic root walls was not considered. For the examined configuration leaflets were assumed to be mounted externally on a supporting stent and modelled as perfectly "glued" over mating surface. The stent is typically significantly stiffer than leaflet tissue and was simply modeled as a linear elastic material with Young modulus of $1900 \mathrm{MPa}$, Poisson ratio 0.4 and density $1320 \mathrm{~kg} / \mathrm{m}^{3}$. For the opening phase valve response was simulated over a period of $0.3 \mathrm{~s}$ according to the law described in Fig. 1. Blood velocity 
at the inlet increased up to a maximum value of $1.2 \mathrm{~m} / \mathrm{s}$ in approximately $0.2 \mathrm{~s}$ and then decreased to zero, while keeping zero pressure boundary condition at the outlet. In the same graph the corresponding inlet flow rate is also reported. Peak flow rate is about $0.5 \mathrm{dm}^{3} / \mathrm{s}$ and cardiac output is about $5.86 \mathrm{l} / \mathrm{min}$ for 71 beats/min, in very good agreement with physiological values.

The solid parts were meshed using 7962 first order tetrahedral elements whereas the fluid domain was discretized with 115091 first order tetrahedral elements. To account for mesh distortion a hyperelastic algorithm for mesh smoothing was adopted, especially suitable for regions where the mesh is highly stretched. However, as already mentioned, in the opening phase mesh deformation could be so severe to compromise solution quality. A remeshing algorithm available in the software was therefore used to remesh fluid domain when element quality fall below a threshold value. To solve the time-dependent simulation a fully-coupled approach was employed, in which the multiple physics are coupled together: fluid velocity and pressure, as well as leaflet and stent motion and stress/strain, are solved at the same time. A direct solver was chosen with a damped version of Newton's methods, imposing a maximum time stepping of $0.5 \mathrm{~ms}$.

\subsection{Leaflet constitutive law}

For the purpose of the present study a key point is the choice of constitutive law for the leaflet and the definition of fiber reinforcement directions. As anticipated leaflets properties and constitutive models were chosen taking as a reference bovine pericardial valves. For this tissue it is common to most recent quasi-static or dynamic structural analyses the adoption of nonlinear elastic constitutive model. ${ }^{15-17}$ In some recent works ${ }^{7,8}$ anisotropic models of the type proposed by Holzapfel ${ }^{18}$, well accepted for modeling biological tissues, have been adopted for BP. In particular according to the approach implemented by Auricchio et al. ${ }^{7}$ the tissue is considered as consisting of a matrix with two family of fibers embedded, symmetrically organized with respect to a preferred fiber direction that corresponds to the base-to-apex direction of the original pericardial sheet and to the circumferential direction for the leaflet in the assembled valve.

The two fiber families are mechanically equivalent (i.e.same material parameters) and are assumed to be arranged with an angle $\beta$ with respect to the preferred orientation. The strain energy function is defined as follows:

$\Psi=c_{10}\left(I_{1}-3\right)+\sum_{i} \frac{k_{1 i}}{2 k_{2 i}}\left\{\exp \left[k_{2 i}\left(I_{4 i}-1\right)^{2}\right]-1\right\}$

In equation (1) $\mathbf{I}_{1}$ is the first invariant of the right Cauchy-Green deformation tensor $\mathbf{C}$ and $\mathbf{I}_{4 \mathrm{i}}$ a pseudo-invariant defined as: 


$$
I_{4 i}=\mathrm{C}: \mathrm{a}_{0 i} \otimes \mathrm{a}_{0 i}
$$

where $\mathbf{a}_{0 \mathrm{i}}$ is a unit vector identifying the $\mathrm{i}^{\text {th }}$ preferred direction. The parameter $\mathrm{c}_{10} \geq 0$ is related to the matrix, parameters $k_{1 \mathrm{i}}, k_{2 \mathrm{i}}>0$ are associated with the response of the $i^{\text {th }}$ collagen fiber. The values of the parameters are $c_{10}=20.1$ $\mathrm{kPa}, \mathrm{k}_{1}=54.62 \mathrm{kPa}, \mathrm{k}_{2}=30.86, \beta=29.8^{\circ}$. Once the strain energy density is defined, the second Piola-Kirchoff stress can be computed as:

$$
S=2 \frac{\partial \Psi}{\partial C}
$$

Interestingly a similar approach, but with a slightly different definition of the strain energy contributions, has been adopted also to model a newly developed polymeric valve. ${ }^{9}$ This anisotropic strain energy formulation well suited the purpose of the present work, since by varying fiber angle $\beta$ in eq. (1) different degree of anisotropy can be obtained, whereas by changing the value of coefficient $\mathrm{c}_{10}$ the influence of matrix can be investigated. The effect of varying fiber orientations can be appreciated in Fig. 2 where equibiaxial response for the case with $\mathrm{c}_{10}=20 \mathrm{kPa}$ is reported.
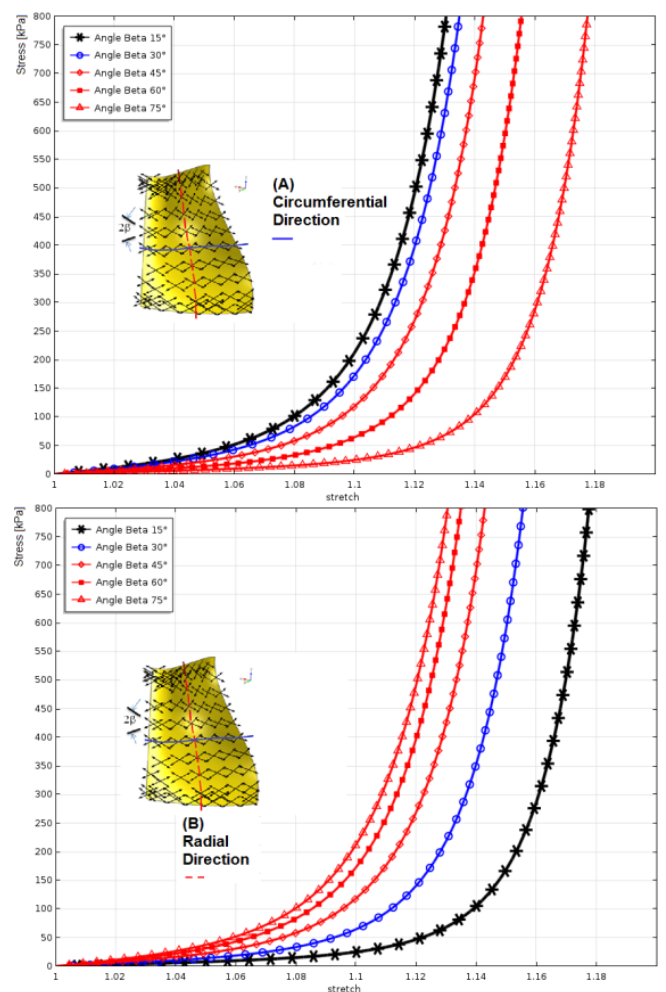

Fig. 2 Tissue equibiaxial stress-strain response as a function of fibers orientations (a) circumferential direction (b) radial direction 
It should be underlined that this type of constitutive law is not directly available as built-in model in Comsol Multiphysics (C) and it was therefore introduced in the code by means of user defined domain variables. The model is implemented in a nearly-incompressible form (bulk modulus 1.0e9 Pa), including in the definition of pseudo-invariants the constraint that fiber contribution is only active when they are stretched in tension.

It should also be noted that the definition of fiber directions in the reference undeformed configuration, in which the leaflet already possessed a curved shape, required a preliminary step of analysis, taking advantage of Partial Differential Equation (PDE) modulus of the software.

\subsection{Case studies}

The valve was first studied in a reference configuration adopting the same material parameters reported in literature. ${ }^{7}$ Then the following case studies were considered:

Case A) $\quad$ Fixed fiber orientation $\left(\beta= \pm 30^{\circ}\right)$

Variable matrix stiffness $\left(10<\mathrm{c}_{10}<200 \mathrm{kPa}\right)$

Case B) Variable fiber orientation $\left(\beta= \pm 15^{\circ}, 30^{\circ}, 45^{\circ}, 60^{\circ}, 75^{\circ}\right)$

Low matrix stiffness $\left(c_{10}=20 \mathrm{kPa}\right)$

Case C) $\quad$ Variable fiber orientation $\left(\beta= \pm 15^{\circ}, 30^{\circ}, 45^{\circ}, 60^{\circ}, 75^{\circ}\right)$

Higher matrix stiffness $\left(c_{10}=100 \mathrm{kPa}\right)$

The influence of matrix stiffness and fiber orientation on valve performances was evaluated considering the following indexes during the cycle:

- Inlet Average Pressure $\left(\mathrm{DP}_{\text {ave }}\right)$

- Effective Orifice Area (EOA)

Note that $\mathrm{DP}_{\mathrm{ave}}$ was computed directly in Comsol Multiphysics, by an averaging operator defined on the inlet section. EOA was computed by means of a script ${ }^{19}$ previously implemented in Matlab@ for digital image analysis, enabling projection of deformed valve mesh on a plane parallel to the annulus for each time increment and automated calculation of corresponding lumen.

Valve performances were also compared at time $0.215 \mathrm{~s}$, when all valves exhibited complete opening, considering:

- Transvalvular pressure distribution along conduct centerline (DP inout $)$

- Blood flow velocity along conduct centerline at time $\left(\mathrm{V}_{\max }\right)$

Note that in following graphs fiber orientation and matrix stiffness may be indicated with a compact notation (i.e. b75_m20 means angle $\beta=75^{\circ}, \mathrm{c}_{10}=20$ $\mathrm{kPa})$ 


\section{Results}

\subsection{Valve leaflet dynamics and blood flow for reference configuration}

Valve behavior was first examined in the reference configuration previously defined. In Fig. 3 valve orifice is represented as seen from aortic side, with contour maps referring to total displacement. The valve reached open configuration after $0.065 \mathrm{~s}$. Then EOA further increased, with some rebounds due to oscillations of the free edge of the leaflet, reaching its peak values in the period $0.15-0.25$ s. Finally, as inlet flow rate decreased, the valve started returning to its initial position.

Overall the model predicted a peak transvalvular pressure $\left(\mathrm{DP}_{\text {ave }}\right)$ of approximately $10.5 \mathrm{mmHg}$ and EOA of $2.97 \mathrm{~cm}^{2}$. Such values compare very favorably with those reported for valves of similar configuration. As an example for a size 27 valve, mean systolic pressure gradient of $12.4 \pm 2.4$ $\mathrm{mmHg}$ and mean EOA of $2.36 \pm 0.4 \mathrm{~cm}^{2}$ have been reported. ${ }^{20}$ Also the leaflets exhibit realistic modes of deformation, compared to in-vitro images. ${ }^{21}$

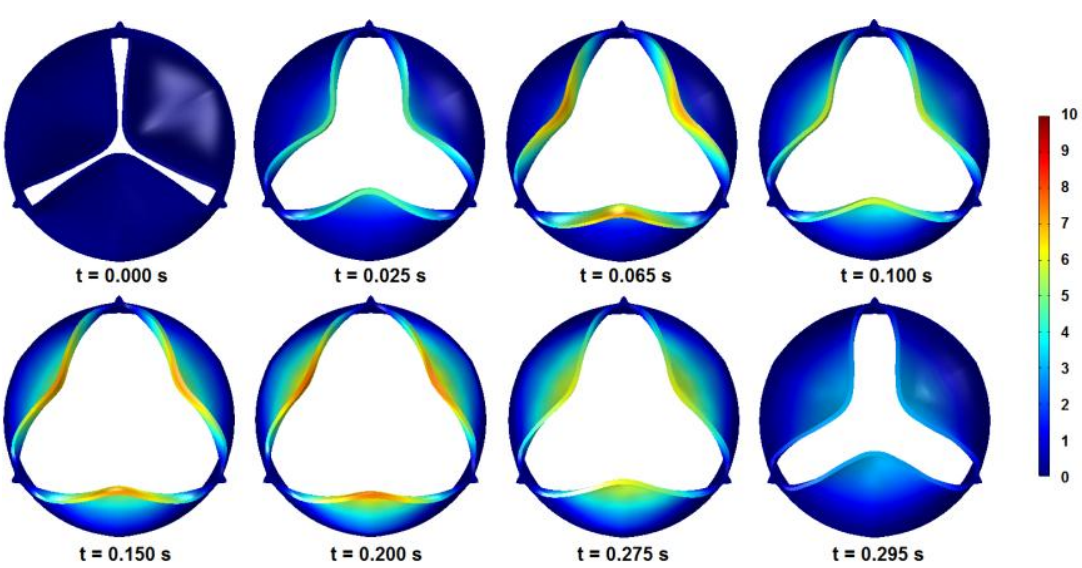

Fig. 3 Progressive valve opening (reference valve), Total displacement [mm]

Blood flow across the valve is represented in Fig. 4. Contour maps refer respectively to blood velocity $(\mathrm{m} / \mathrm{s})$ in the mid-leaflet symmetry section and to pressure exerted $(\mathrm{mmHg})$ by the fluid on the leaflet surface.

During the opening phase the progressive formation of a central jet-like flow can be clearly noticed. Blood flow at valve downstream reached a peak value of $\mathrm{V}_{\max }$ about $2 \mathrm{~m} / \mathrm{s}$ (with some noticeable oscillation in the peak opening period), again in line with values reported for this type of valve. ${ }^{22}$ Overall it can be concluded that in presence of a physiological cardiac flow rate, the valve operates with values of $\mathrm{EOA}, \mathrm{DP}_{\mathrm{ave}}$ and $\mathrm{V}_{\max }$ in very good agreement with those reported in literature. For the purpose of this study the model could thus be used as a base to evaluate changes related to the material properties. Finally 
it should be mentioned that the stress state showed a maximum peak in the commissural region of approximately 0.1 MPa. Since this value is lower that those typically reported for the closure phase and did not vary significantly for the case studies considered, results were not reported. ${ }^{19}$
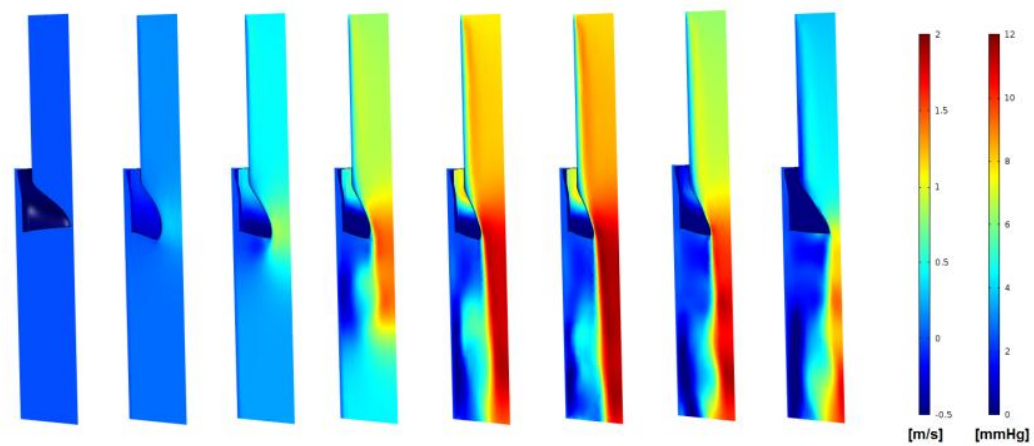

$t=0.000 s \quad t=0.065 s$

$t=0.100 \mathrm{~s} \quad t=0.150 \mathrm{~s}$

$t=0.200 \mathrm{~s} \quad t=0.225 \mathrm{~s}$

$t=0.275 \mathrm{~s}$

$\mathrm{t}=0.295 \mathrm{~s}$

Fig. 4 Flow conditions, reference valve: Velocity $(\mathrm{m} / \mathrm{s})$, Surface pressure $(\mathrm{mmHg})$

\subsection{Case study A}

For the case study A the fiber orientation is the same as the reference model (i.e. $\beta=30^{\circ}$ ) and matrix stiffness is modified by changing the value of parameter $\mathrm{c}_{10}$ in the range $10-200 \mathrm{kPa} . \mathrm{DP}_{\text {ave }}$ and EOA are reported as a function of time in Fig. 5(a,b).

The influence of matrix stiffening is quite apparent since in the worst case scenario, when a value of $200 \mathrm{kPa}$ is assumed for $\mathrm{c}_{10}, \mathrm{DP}_{\mathrm{ave}}$ may increase up to $20 \mathrm{mmHg}$ and EOA may decrease down to $2.10 \mathrm{~cm}^{2}$.

Matrix stiffness also clearly influence opening times and dynamics. For low matrix stiffness $(10-20 \mathrm{kPa})$ the valve reaches a fully open configuration in about $0.05-0.075 \mathrm{~s}$ and then exhibits a sort of rebound before reaching again a maximum, as a result of leaflet higher flexibility.

On the contrary for higher matrix stiffness valve opening is slower and more progressive. Note that opening times reported in literature are quite variable, depending on measurement conditions or modeling assumptions.

Present values are similar to those reported by Ranga et al. ${ }^{12}$ as physiological, but comparatively higher than those reported in other studies (typically about $0.02 \mathrm{~s}) .^{16,19,23}$

Considering now time $\mathrm{t}=0.215 \mathrm{~s}$ as a reference time for valve comparison in a fully open condition, in Fig 5 (c,d) the distribution of pressure and velocity as a function of $\mathrm{z}$-axis coordinate along the centerline of the conduct can be appreciated.

Results show that locally the transvalvular pressure gradient across the valve (i.e. pressure difference between leaflet ventricular and aortic surfaces) is 
rather steep and pressure applied at different valve heights from the annulus plane (located at $\mathrm{z}=0 \mathrm{~m}$ ) changes significantly.

By increasing matrix stiffness also the slope of transvalvular pressure curve increased. Considering instead peak velocity, blood flow at valve downstream ranges between about $1.75 \mathrm{~m} / \mathrm{s}$ for low matrix stiffness and average values up to $2.5 \mathrm{~m} / \mathrm{s}$ for stiffer matrix, in which case also noticeable oscillation can be observed moving toward the outlet section.

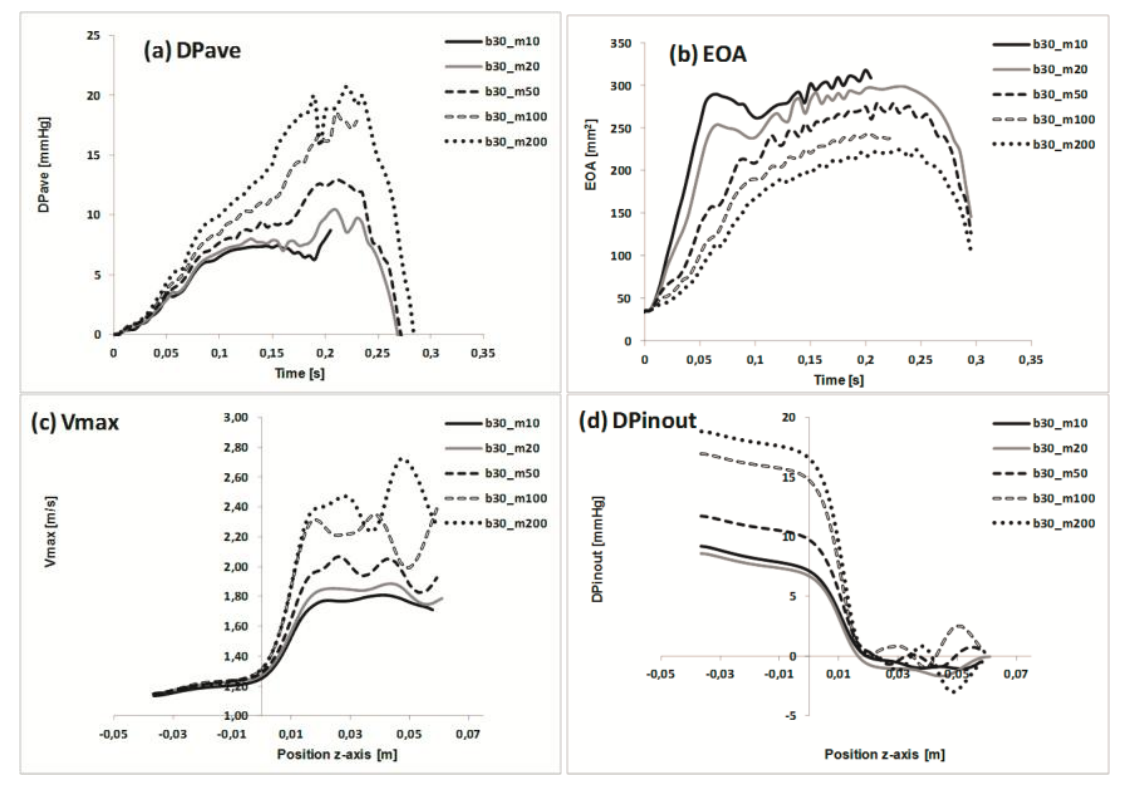

Fig. 5 Case A, (a)DP ${ }_{\text {ave }},\left(\right.$ b)EOA, (c) DP inout, $_{\text {, }}$ (d) $\mathrm{V}_{\text {max }}$

\subsection{Case study B}

$\mathrm{DP}_{\text {ave }}, \mathrm{EOA}$ are reported as a function of time in Fig. $6(\mathrm{a}, \mathrm{b})$ for the case study $B$, in which the orientation of the two fiber families is varied with respect to the circumferential direction of the valve, while keeping the same matrix stiffness as reference model (i.e. $\mathrm{c}_{10}=20 \mathrm{kPa}$ ).

In the first phase of the opening process the influence of fiber orientation is initially modest: opening times appear to be controlled by the chosen matrix stiffness rather than fiber directions. On the contrary, once the valve is open fiber orientation has a major influence on peak value of both $\mathrm{DP}_{\text {ave }}$, EOA. Valve configurations having fiber more aligned towards the radial direction (i.e higher values of $\beta$ angle) exhibit EOA up to $3.7 \mathrm{~cm}^{2}$ and correspondingly operates with lower pressure gradients.

Similar considerations apply considering again results at time $t=0.215 \mathrm{~s}$ along the conduct, reported in Fig. 6(c,d). Decreasing stiffness in the circumferential direction leads to significantly diminution of $\mathrm{V}_{\max }$ and $\mathrm{DP}_{\text {inout }}$, apparently 
resulting, at least for the opening phase, in effects similar to a matrix stiffness decrease.

Basing on this result, it can be concluded that once the valve is open, the maximum EOA potentially achievable is controlled by the stiffness in the circumferential direction.

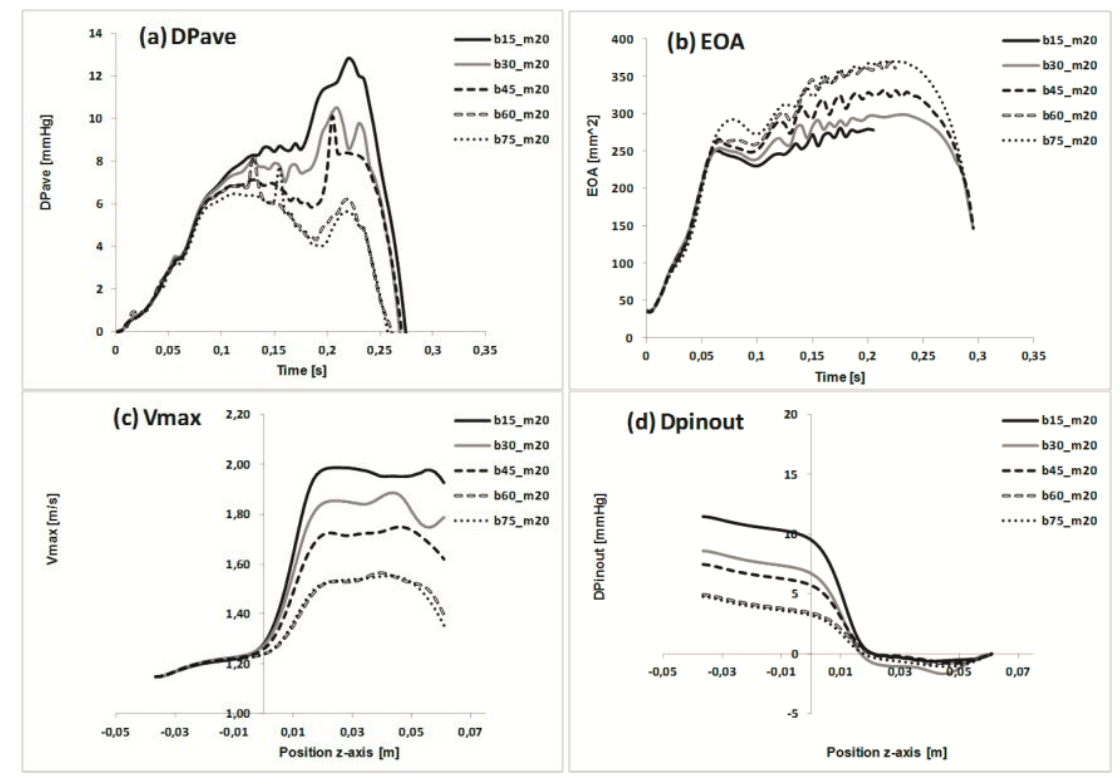

Fig. 6 Case A, (a)DP ave, (b)EOA, (c) DP inout $_{\text {, }}(\mathrm{d}) \mathrm{V}_{\max }$

\subsection{Case study C}

$\mathrm{DP}_{\mathrm{ave}}$, EOA are reported as a function of time in Fig. 7 (a,b) for the case study $\mathrm{C}$, in which the orientation of the two fiber families is varied with respect to the circumferential direction of the valve, while imposing higher matrix stiffness (i.e. $\mathrm{c}_{10}=100 \mathrm{kPa}$ ).

Overall results present similar trends as for case B, but some important differences can be noticed. In particular, considering the influence on EOA of varying fiber angle $\beta$, a limited effect can be appreciated, except for the case in which the lowest fiber angle of $15^{\circ}$ is adopted (i.e. highest circumferential stiffness). In this case a value of EOA of about $2.1 \mathrm{~cm}^{2}$ was observed and valve operated with higher values of transvalvular pressure which in turn resulted, as clearly noticeable comparing Fig. 6(d) and Fig. 7(d), in downstream fluctuation of blood velocities. 


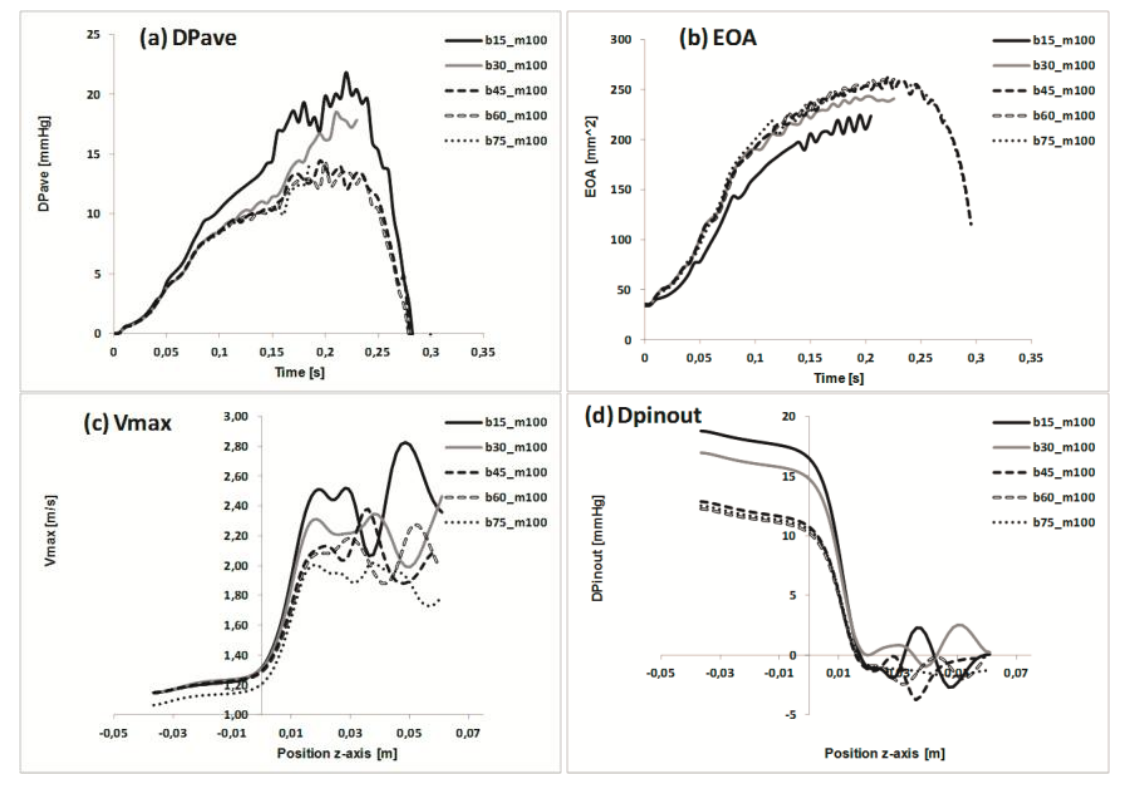

Fig. 7 Case A, (a)DP ave, (b)EOA, (c) $\mathrm{DP}_{\text {inout, }}$ (d) $\mathrm{V}_{\text {max }}$

\section{DISCUSSION AND CONCLUSIONS}

Basing on the results reported in the previous paragraph some observations should be remarked.

\subsection{Influence of matrix stiffness and fiber orientation}

Matrix stiffness and fiber orientation have a pronounced effect on valve functional performances, in particular considering clinically relevant indexes such EOA and transvalvular pressure gradient. The effects of fiber orientation may be amplified in presence of very soft matrix, whereas an increase of matrix stiffness may mask such effect. By varying matrix stiffness only, significant variations of performances could be observed, leading in some case to rather high pressure gradient. As a consequence these should be regarded as important design parameters to be taken into account.

During the opening stage, and in particular after the initial transition from closed to open configuration, for the valve examined higher values of EOA were found the more the preferred fiber direction (i.e. with lower extensibility) was assumed radially (i.e. from stent annulus towards leaflet free edge) rather than circumferentially. Maximum achievable EOA seems to be controlled by the expansion of the leaflet on planes transversal to the axial direction, and therefore higher values of $\beta$ angle are more favorable. This is however in contrast with findings reported in other investigations in which, basing on structural only analyses but including also the closure phase, it was concluded 
that a preferred circumferential alignment of collagen fibers could be beneficial for pericardial $\mathrm{BHV}$, with particular reference to stress state. ${ }^{17,24}$ On the other hand Liu et al. ${ }^{25}$ found that for a single ply fiber-reinforced composite leaflet model, an orientation of the fibers at $\pm 30^{\circ}$ resulted in a minimization of the maximum principal stress in the polymer matrix and that embedding fibers perpendicular to the intersection edge to the stent would reduce the leaflet stress. For the closure phase stress state and coaptation may be effectively studied using "dry" models. Previous study on the same type of valve showed that by following the principle that fiber should be aligned with principal loading directions, complex architecture could be necessary, to account for different directions of principal stress and strain directions in the commissural region and near valve ring. ${ }^{19}$

Furthermore by aligning the fiber "radially" the leaflet exhibited higher displacements in the axial directions, suggesting that in combination with low stiffness matrix, leaflet coaptation process may potentially be impaired.

While in this sense a compromise solution could be possibly to adopt a $\pm 45^{\circ}$ configuration for the fiber families, overall results confirm that the optimization of leaflet properties remains a challenging task, in which it is necessary to balance contrasting effects and to consider both structural and functional aspects.

\subsection{Modeling aspects}

A part from sensitivity of valve performances to investigated parameters, the model provided the opportunity to consider some relevant aspects from a modeling point of view.

First of all different approaches can be found in literature to reproduce the mechanical behavior of the leaflets. As an example linear elastic isotropic response has often been adopted, especially in the context of FSI investigation due to inherent complexity of the models. ${ }^{10,26}$

Comparative structural analyses on natural and bioprosthetic valves have already underlined the importance of considering tissue anisotropy, ideally within the framework of hyperelasticity theory to take into account large deformation behavior. ${ }^{27,28}$

Under this point of view the inclusion of an anisotropic hyperelastic law in a FSI model is thus a relevant point addressed by the present study. Overall results also shows that when considering functional aspects special care should be exercised when choosing constitutive models and fitting associated parameters: by varying matrix stiffness from 10 to $200 \mathrm{kPa}$, variations of EOA up to $50 \%$ could be observed.

Another important aspect is that significant differences in the pressure gradients across the two sides of the valve were present for different valve heights. In particular the region near the annulus is subjected to higher pressure compared to leaflet free edge both in the initial phase and both when valve is fully open. This clearly put into evidence some limit of structural-only 
analysis, in which the action of the fluid is transferred to the leaflet as a spatially and temporally uniform pressure. This acts as a follower load throughout the cycle, possibly leading to underestimation of opening times and influencing deformation patterns and stress level.

\subsection{Study limitations and future developments}

Any model of prosthetic valve operating into a complex biological environment necessarily embodies some simplifications and limitations.

From a fluid dynamics point of view, in the region of the aortic valve turbulent flow does occur, but it is still very difficult to recreate it computationally as there is very limited availability of direct turbulence measurements. Although recently LES turbulence model were adopted for investigations on $\mathrm{BHV},{ }^{29} \mathrm{a}$ laminar flow was assumed, as in some previous investigations on prosthetic valves. ${ }^{10}$ Here the main purpose of including interaction with fluid was to create a model of the valve capable to reproduce loading conditions more realistically than structural only approach. In reality blood flows across prosthetic heart valves are extremely complex (pulsatile, borderline turbulent and transitional) and in general resolving all hemo-dynamically relevant length scales of motion under physiological conditions would require very fine mesh and massive parallel computing resources. ${ }^{30}$

Ideally the model should also cover both opening and closure phases and in this case it could also be necessary to consider the compliance of aortic root wall and annulus. In the present work FSI simulation of the closure phase has not been included in the model, mainly due to inherently high difficulties with the ALE method, as available in the software, to cope with topological changes of fluid domain that may occur during the closure phase as a result of leaflet coaptation.

Finally the results were compared with literature findings on similar confuguration but of course a complete validation of the model would require an experimental campaign to test real valve on examined parameters.

In conclusion valve hemodynamics and leaflet properties are currently tested by manufacturers, but there is a constant effort towards a continuous improvement of their performances. This is especially true for biological valves, for which not all aspects of structural response and interaction with biological environment can be considered completely understood. In this context, the present study may provide useful information for those working in the field of leaflets' material selection. In particular, results could be relevant for novel tissue engineered leaflets, in which case fiber direction and matrix stiffness can actually be tailored and tuned for specific goals.

Of course the process of designing new valves is complex and highly time/money consuming and any change to configurations currently authorized for use by regulatory bodies, must be very carefully evaluated. The influence on clinical practice of present findings should therefore be evaluated in the long period rather than as an immediate outcome. Furthermore, experimental 
and clinical evidences show that for BHV the degree of anisotropy and extensibility may also change considerably because of calcification processes or cyclic loading and damage models have been recently proposed to reproduce this effect ${ }^{31,32,33}$. The combination of present FSI approach with such models could help in the future to gain better understanding on the effects of such processes on performance variations and safety critical aspects during valve lifetime.

\section{References}

1. Mohammadi H, Mequanint K, Prosthetic aortic heart valves: Modeling and design, Med Eng Phys, 33(2):1331-1347, 2001.

2. Sacks MS, Chuong CJ. Orthotropic mechanical properties of chemically treated bovine pericardium, Ann Biomed Eng, 26:892-902, 1998.

3. Zioupous P, Barbenel JC, Fisher J, Anisotropic elasticity and strength of glutaraldehyde fixed bovine pericardium for use in pericardial bioprosthetic valves, $J$ Biomed Mater Res. 28(1):49-57, 1994.

4. Langdon SE, Cherneky R, Pereira CA, Abdulla D, Lee JM, Biaxial mechanical/structural effects of equibiaxial strain during crosslinking of bovine pericardial xenograft materials, Biomaterials, 20:137-153,1999.

5. Ghanbari H, Viatge H, Kidane AG, Burriesci G, Tavakoli M, Seifalian AM, Polymeric heart valves: New materials, emerging hopes, Trends Biotechnol, 27(6):359$367,2009$.

6. Claiborne TE, Slepian MJ, Hossainy S, Bluestein D, Polymeric trileaflet prosthetic heart valves: evolution and path to clinical reality, Expert Rev Med Devices, 9(6):577594,2012

7. Auricchio F, Conti M, Ferrara A, Morganti S, Reali A Patient-Specific simulation of stentless aortic valve implant: the impact of fibres on leaflet performance, Comput Meth Biomech Biomed Eng, 17(3):277-285, 2014

8. Martin C, Sun W, Bio-prosthetic heart valve stress analysis: impact of leaflet properties and stent tip deflection, in Proulx T (ed.), Mechanics of biological Systems and Materials Vol. 2 - Proceedings of the 2011 SEM annual conference on Experimental and Applied Mechanics, Springer, Berlin Heidelberg New York, pp. 7378,2011

9. De Gaetano F, Bagnoli P, Zaffora A, Pandolfi A, Serrani M, Brubert J, Stasiak J, Moggridge GD, Costantino ML, A newly developed tri-leaflet polymeric heart valve prosthesis, J Mech Med Biol, 15(2):1540009-1-17, 2015

10. Van Aswegen KHJ, Smuts AN, Scheffer C, Investigation of leaflet geometry in a percutaneous aortic valve with the use of fluid-structure interaction simulation. $J$ Mech Med Biol 12(1):125003-1-15, 2012

11. De Hart J, Baaijens FPT, Peters GWM, Schreurs PJG, A computational fluidstructure interaction analysis of a fiber reinforced stentless aortic valve. J Biomech 36:699-712, 2003 
12. Ranga A, Bouchot O, Mongrain R, Ugolini P, Cartier R, Computational simulations of the aortic valve validated by imaging data: evaluation of valve sparing techniques. Interact Cardiovasc Thorac Surg, 5:373-378, 2006.

13. Marom G, Haj-Ali R, Raanani E, Schafers HJ, Rosenfeld M, A fluid-structure interaction model of the aortic valve with coaptation and compliant aortic root, Med Biol Eng Comput, 50:173-182, 2012

14. Comsol Multiphysics AB, Structural Mechanics Module User's Guide: Theory for the Fluid-Structure Interaction Interface, 2015

15. Kim H, Lu J, Sacks MS, Chandran KB, Dynamic simulation of pericardial bioprosthetic heart valve function. J Biomech Eng, 128(5):717-24, 2006.

16. Saleeb AS, Kumar A, Thomas VS. The important roles of tissue anisotropy and tissue-to-tissue contact on the dynamical behavior of a symmetric tri-leaflet valve during multiple cardiac pressure cycles, Med Eng Phys, 35(1):23-35, 2013

17. Li K, Sun W Simulated thin pericardial bioprosthetic valve leaflet deformation under static pressure-only loading conditions: implications for percutaneous valves, Ann Biomed Eng, 38(8):2690-2701, 2010

18. Holzapfel GA, Gasser TC, Ogden RW, A new constitutive framework for arterial wall mechanics and a comparative study of material models, J Elasticity, 61:1-48, 2000.

19. Avanzini A, Battini D, Structural analysis of a stented pericardial valve with leaflets mounted externally, Proc Inst Mech Eng H, 228(10):985-995, 2014.

20. Bleizifer S, Eichinger WB, Hettich IM, Ruzicka D, Badiu CC, Guenzinger R, Bauernschmitt R, Lange R, Hemodynamic characterization of the Sorin Mitroflow Pericardial Bioprosthsis at rest and exercise, J Heart Valve Dis, 18:95-100, 2009

21. Jennings ML, El-Gatit A, Nagy ZL, Fisher J, Walker PG, Watterson KG. Hydrodynamic function of the second-generation mitroflow pericardial bioprosthesis. Ann Thorac Surg, 74:63-68, 2002.

22. Diab M, Faerber G, Bothe W, Lemke S, Breuer M, Walther M, Doenst T, Sizing strategy is a major determinant of postoperative pressure gradients in commonly implanted stented tissue valves, Eur J Cardiothorac Surg, 44(4):1-6, 2013

23. Watton P, Luo X, Wang X, Bernacca G, Molloy P, Wheatley D. Dynamic modelling of prosthetic chorded mitral valves using the immersed boundary method. J Biomech, 40:613-26, 2007

24. Arcidiacono G, Corvi A, Severi T. Functional analysis of bioprosthetic heart valves. J Biomech, 38(7): 1483-1490, 2005.

25. Liu Y, Kasyanov V, Schoephoerster RT, Effect of fiber orientation on the stress distribution within a leaflet of a polymer composite heart valve in the closed position, $J$ Biomech, 40:1099-1106, 2006

26. Hsu MC, Kamensky D, Bazilevs Y, Sacks MS, Hughes TJR, Fluid-structure analysis of bioprosthetic heart valves: significance of arterial wall deformation, Comput Mech, 54(4):1055-1071, 2014 
27. Patterson EA, Howard IC and Thornton MA. A comparative study of linear and nonlinear simulations of the leaflets in a bioprosthetic heart valve during the cardiac cycle. J Med Eng Technol, 20(3): 95-108, 1996

28. Koch TM, Reddy BD, Zilla P, Franz T Aortic valve leaflet mechanical properties facilitate diastolic valve function, Comput Methods Biomech Biomed Engin, 13(2):225234,2010

29. Sirois E Sun W, Computational Evaluation of Platelet Activation Induced by a Bioprosthetic Heart Valve, Artil Organs, 35(2):157-165, 2010.

30. Yoganathan AP, Chandran KB, Sotiropoulos F, Flow in prosthetic heart valves: State-of-the-art and future directions, Ann Biomed Eng 33(12):1689-16942005

31. Sun W, Sacks M, Fulchiero G, LoveKamp J, Vyavahare N, Scott M, Response of heterograft heart valve biomaterials to moderate cyclic loading, J Biomed Mater Res Part A, 69A(4):658-669, 2004

32. Martin C, Sun W, Modeling of long-term fatigue damage of soft tissue with stress softening and permanent set effects, Biomech Model Mechanobiol, 12:645-655, 2013

33. Martin C, Sun W, Simulation of long-term fatigue damage in bioprosthetic heart valves: effects of leaflet and stent elastic properties, Biomech Model Mechanobiol, 13(4):759-770, 2014 